\title{
Geschichte machen in Frankreich - Einleitung
}

Falk Bretschneider, Mareike König et Pierre Monnet

\section{(2) OpenEdition}

Journals

Édition électronique

URL : http://journals.openedition.org/ifha/7834

DOI : $10.4000 /$ ifha.7834

ISSN : 2198-8943

Éditeur

IFRA - Institut franco-allemand (sciences historiques et sociales)

Référence électronique

Falk Bretschneider, Mareike König und Pierre Monnet, « Geschichte machen in Frankreich - Einleitung », Revue de l'IFHA [Online], HS | 2014, Online erschienen am: 01 September 2014, aufgerufen am 22 September 2020. URL : http://journals.openedition.org/ifha/7834 ; DOI : https://doi.org/10.4000/ifha. 7834

Ce document a été généré automatiquement le 22 septembre 2020.

CC BY-SA 3.0 


\title{
Geschichte machen in Frankreich - Einleitung
}

\author{
Falk Bretschneider, Mareike König et Pierre Monnet
}

Im Jahr 2011 erschien auf perspectivia.net - der internationalen elektronischen Publikationsplattform der unter dem Dach der Max Weber Stiftung zusammengefassten deutschen geisteswissenschaftlichen Institute im Ausland - ein Wegweiser für Nachwuchswissenschaftlerinnen und -wissenschaftler mit dem Titel »Faire de l'histoire en Allemagne ${ }^{1}$. Diese Initiative wurde seinerzeit - sowohl redaktionell als auch institutionell - vom Deutschen Historischen Institut Paris (Mareike König) und dem deutsch-französischen Programm der Fondation Maison des sciences de l'homme (Falk Bretschneider) getragen. Das Echo, das sie beim Publikum gefunden hat, war überaus positiv und hat beide Institutionen ermutigt, sich mit dem Institut français d'histoire en Allemagne in Frankfurt a. M. (Pierre Monnet) zusammenzutun und auf diesem Weg nun gewissermaßen das deutsche Pendant zu dieser ersten Publikation vorzulegen: den Forschungswegweiser »Geschichte machen in Frankreich» .

Drei Dinge haben dieses Unternehmen erheblich erleichtert: die bei der ersten Veröffentlichung gewonnenen Erfahrungen, das dabei versammelte savoir-faire von Herausgeberteam sowie Autorinnen und Autoren, vor allem aber die vielen Reaktionen, die von Seiten der Leserinnen und Leser kamen. Über die Ähnlichkeit der Titel hinaus teilen beide Wegweiser auf inhaltlicher Ebene deshalb viele Gemeinsamkeiten. Wer will, kann die vorliegende Publikation somit als ein Spiegelbild des wissenschaftlichen, methodologischen und forschungspraktischen Dialogs sehen, der sich seit Jahrzehnten zwischen Historikerinnen und Historikern auf beiden Seiten des Rheins entwickelt hat.

Geprägt wird dieser Austausch von Nachahmungen und Transfers - und auch die beiden Wegweiser stehen in einem solchen Verhältnis zueinander. Sie sind Teil einer guten Praxis, die darin besteht, aufzunehmen und nachzumachen, was sich beim Nachbarn bewährt hat. Der Wegweiser »Geschichte machen in Frankreich« ist deshalb nicht einfach nur eine Gebrauchsanweisung der französischen Geschichtsforschung für deutschsprachige Geschichtswissenschaftlerinnen und -wissenschaftler. Sondern er ist selbst ein deutsch-französisches Produkt, d.h. er ist gleichermaßen Multiplikator wie 
Grundlage einer Beziehung, die so eng ist, wie nur wenige andere in Europa oder der Welt. Einige wenige Beispiele (unter vielen anderen) mögen genügen, um sich davon zu überzeugen: das deutsch-französische Geschichtsbuch für die drei Klassen der gymnasialen Oberstufe ${ }^{2}$, die in Fertigstellung begriffene elfbändige wissenschaftliche deutsch-französische Geschichte ${ }^{3}$,die Existenz von deutschen historischen Forschungszentren in Paris ${ }^{4}$ bzw. französischen in Berlin ${ }^{5}$ und Frankfurt a. M. ${ }^{6}$ oder die zahlreichen Doktorandenkollegs oder integrierten Studiengänge in Geschichte, die zwischen beiden Ländern von der Deutsch-Französischen Hochschule getragen werden ${ }^{7}$. Durchaus bezeichnend ist, dass auf allen diesen Feldern Historikerinnen und Historiker eine Pionierrolle gespielt haben. Damit führen sie die Tradition einer Disziplin fort, die - seitdem sie sich im 19. Jahrhundert als akademisches Fach etabliert hat - ein zentraler Schauplatz für die Konstruktion von Bildern des jeweils anderen war und ist ... im besten wie im schlimmsten Sinne.

Von alledem wird auch in diesem Wegweiser die Rede sein. Die Leserinnen und Leser werden hier zahlreiche Informationen $\mathrm{zu}$ Austauschbeziehungen, Institutionen, Methoden und Sprachen finden. Daneben wird eine Praxis der Interkulturalität thematisiert, die - obwohl es deutschen und französischen Historikerinnen und Historikern seit Langem zur guten Gewohnheit geworden ist, miteinander in den Dialog zu treten - nach wie vor für manche verwirrend ist. Auch um die z.T. erheblichen Unterschiede zwischen den beiden Hochschulsystemen wird es gehen und um die Art und Weise, wie Geschichtswissenschaftlerinnen und -wissenschaftler auf beiden Seiten des Rheins schreiben, lehren, recherchieren, ihre Quellen erkunden, lesen, nachdenken, publizieren oder auch im weiten Berufsfeld des historischen Faches Karriere machen.

Um alle diese Themen zu bearbeiten, verstand es sich von selbst, dass wir erneut eine deutsch-französische Mannschaft an Autorinnen und Autoren versammelt haben. Eingeladen wurden dafür Spezialistinnen und Spezialisten, die sich nicht nur durch ihre Kenntnisse und Kompetenzen im deutschen und französischen Wissenschaftsbetrieb auszeichnen, sondern im Laufe ihrer Karriere oft auch selbst Forschungserfahrung im anderen Land gesammelt haben. Sie kennen Bibliotheken und Archive in beiden Ländern, schreiben in der und übersetzen aus der anderen Sprache oder betreuen Forschungsarbeiten von Studierenden und Doktoranden in grenzüberschreitenden Studien- und Ausbildungsgängen. Gemeinsamkeiten und Unterschiede im Übergang von einer Forschungslandschaft zur anderen haben sie so alle am eigenen Leib erfahren: Dazu gehören die im Vergleich zu Deutschland spezifische Zweigleisigkeit und komplexe Aufeinanderschichtung von universitären und außeruniversitären Strukturen und Institutionen der Forschung in Frankreich (Pierre Monnet), die unterschiedlichen Rhythmen und Aktualitätsbezüge der gerade als in oder out geltenden Forschungsthemen, die auch eine andere Stellung des Historikers in der Gesellschaft widerspiegeln (Nicolas Offenstadt), die komplexen und stark hierarchisierten Rekrutierungsmechanismen, die sich zwischen den Universitäten einerseits und den außeruniversitären Hochschul- und Forschungseinrichtungen andererseits erheblich unterscheiden (Jean-Louis Georget) oder, dem noch vorgeschaltet, die besonderen Schwierigkeiten, wie sie durch die Institution und die Kultur der durch und durch »französischen« Auswahlverfahren im Bildungsbereich hervorgebracht werden (Claire Gantet).

Diese spezifischen Merkmale unterliegen jedoch einem permanenten Wandel, der sich am Rhythmus der Reformen in Frankreich bemisst, aber auch - und zwar stärker noch 
- an den europäischen Harmonisierungsprozessen und ihrem Einfluss auf Inhalt und Dauer des Studiums (Ulrike Krampl) oder der Promotion (Falk Bretschneider und Christophe Duhamelle), an den neuen Formen und Medien des Publizierens (Anne Baillot und Mareike König) und der Recherche im Internet (Mareike König und Annette Schläfer) oder an den sich wandelnden Herausforderungen des akademischen und nicht akademischen Arbeitsmarkts (Jean-Louis Georget). Jede Autorin und jeder Autor musste also ein Gleichgewicht in einem Spannungsfeld finden: Auf der einen Seite stehen jene Elemente, die auch heute noch als französische Besonderheiten - von einem Modell wird man kaum noch sprechen können - beim Studieren und Schreiben oder "Machen" von Geschichte gelten können, und auf der anderen finden sich die allbekannten Tendenzen einer immer stärker wirksamen internationalen Angleichung von Normen und Praktiken - sei es auf der Ebene von Bibliotheken (Mareike König und Dominique Bouchery) und Archiven (Florence de Peyronnet-Dryden und Mathias Nuding) oder jener der Forschungsfinanzierung (Christiane Schmeken und Delia Küsgen) bzw. der praktischen Fragen eines Forschungsaufenthalts im anderen Land (Niels F. May).

Natürlich gibt es nach wie vor eine »französische Kunst« des Verfassens und der Verteidigung einer Dissertation ${ }^{8}$. Auch wer einen Vortrag vor akademischem Publikum hält (was nach bestimmten rhetorischen Fähigkeiten verlangt) oder einen wissenschaftlichen Artikel für französische Zeitschriften verfasst (Anne Baillot und Franziska Heimburger) ${ }^{9}$, kommt um die Kenntnis von kulturellen Besonderheiten nicht herum. Die französische Tradition zeichnet sich zudem durch einen eigenen und mitunter recht gewöhnungsbedürftigen Stil des Seminars oder der Vorlesung aus. Und das ist auch gut so. Denn eine bloße Angleichung der Formen und Inhalte der Wissensproduktion wäre in Europa - einem Kontinent, dessen Gedeihen oder Verderben davon abhängt, ob und wie er mit Diversität, kultureller Vielfalt und Mehrsprachigkeit zurechtkommt - ein echtes Armutszeugnis.

Selbstverständlich gibt es in Frankreich nach wie vor auch noch die erdrückende Dominanz der Region Paris, die den Forschungsstrukturen im ganzen Land einen nachhaltigen Stempel aufdrückt und den Mittelfluss im gesamten Forschungssektor bestimmt - auch in Bereichen wie den historischen Museen (Judith Dehail) oder der Verbreitung von wissenschaftlichen Büchern und Zeitschriften (Juliette Guilbaud). Um die herrschenden Konvergenzen und Divergenzen zu entschlüsseln, mussten alle Autorinnen und Autoren vor allem aber über ihr jeweiliges Spezialgebiet hinausgehen und den Blick mit bedenken, den ihre Leserinnen und Leser auf Themen werfen, die ihnen mal fremd und mal vertraut vorkommen werden.

Allen Autorinnen und Autoren möchten wir an dieser Stelle sehr herzlich dafür danken, dass sie sich auf diese Herausforderung eingelassen und der gleichermaßen kritischen wie heilsamen Übung ausgeliefert haben, den Blick nicht nur auf den anderen, sondern auch auf sich selbst zu richten. In einer Zeit, in der sich die französischen Historikerinnen und Historiker über den Sinn, den Gegenstand, die Zukunft und die gesellschaftliche Anerkennung ihrer Disziplin erhebliche Gedanken machen ${ }^{10}$, erscheint uns dies noch bemerkenswerter.

In diesem Sinne reiht sich der vorliegende Wegweiser durchaus in einen größeren Reflexionsprozess ein, für den Forschungsprozesse und Forschungsergebnisse - mögen sie sich auch mit Gegenständen befassen, die (wie in der Geschichtswissenschaft die Regel) zeitlich ein gutes Stück zurückliegen - untrennbar mit dem zeitgenössischen 
Umfeld verbunden sind, in dem sie sich entfalten. Sicher ist dieses Umfeld zunächst ein intellektuelles ${ }^{11}$.Es wird aber auch durch alltagspraktische, finanzielle, materielle und psychologische Faktoren geprägt, die in ihren historisch wandelbaren Erscheinungsformen zu analysieren Geistes- und Sozialwissenschaftler nicht nur die Chance, sondern zweifellos sogar die Pflicht haben ${ }^{12}$. Auch das gehört zu dem, was man links wie rechts des Rheins das "Handwerk « der historischen Zunft nennt ${ }^{13}$.

In dieser Perspektive wagen wir sogar zu hoffen, dass der Wegweiser über den Kreis der deutschsprachigen Historikerinnen und Historiker hinaus, an den er sich natürlich zuerst richtet, auch für Spezialisten anderer benachbarter Disziplinen interessant sein könnte, die mit der Geschichtswissenschaft die historische Gebundenheit ihrer Forschungsobjekte, Diskurse und Ansätze teilen. Und vielleicht hilft er sogar französischen Studierenden, Hochschullehrenden und Forschenden selbst, sich im eigenen System besser zurechtzufinden und - in einer Epoche, die durch permanente und grundsätzliche Neustrukturierungen der Schul-, Hochschul- und Wissenschaftslandschaft gekennzeichnet ist - ihre Denk- und Arbeitsumgebung besser zu verstehen. Denn so verhält es sich oft in diesem Spiel aus Transfer und Austausch, mit dem dieses Vorwort begonnen hat und das die Urheberinnen und Urheber dieses Wegweisers immer wieder inspiriert hat: der Umweg über den anderen hilft, sich selbst besser kennenzulernen ...

Bevor wir das Wort nun den Autorinnen und Autoren der einzelnen Artikel überlassen, zwischen denen sich die Leserinnen und Leser natürlich ihren eigenen Leseweg bahnen mögen, möchten wir noch auf die große Unterstützung hinweisen, die dieser Wegweiser von den Institutionen erfahren hat, die ihn produziert, finanziert und gefördert haben: das Deutsche Historische Institut Paris, die Fondation Maison des sciences de l'homme und das Institut français d'histoire en Allemagne in Frankfurt a. M. Manche Beiträge wurden gleich auf Deutsch verfasst, andere hingegen auf Französisch - sie mussten also übersetzt werden, was bekanntlich eine nächste Übung im interkulturellen Transfer ist. Ohne die Übersetzungsarbeit von Tim Geelhaar, Niels F. May, Philipp Siegert und Volker Zimmermann, aber auch ohne das sorgfältige und unverzichtbare Lektorat und den Textabgleich durch Ann Catrin Apstein-Müller hätte dieser Wegweiser nicht das Licht der Welt erblickt. Ein besonderer Dank gilt schließlich der Deutsch-Französischen Hochschule, die im Rahmen der Förderung des DeutschFranzösischen Doktorandenkollegs »Unterschiede denken: Strukturen - Ordnung Kommunikation« (ein Titel, der dem Geist dieses Wegweisers ganz und gar entspricht) die Herstellung dieser Publikation großzügig unterstützt hat. Sie erfüllt so ihre zentrale Mission, unsere beiden universitären, wissenschaftlichen und akademischen Welten einander noch näher zu bringen.

Verfügbar ist dieser Wegweiser ab sofort auf dem elektronischen Portal, das das Institut français d'histoire en Allemagne im Jahr 2013 auf der Plattform revues.org eingerichtet hat. Hier finden sich auch die Rezensions-Datenbank und die verschiedenen Ausgaben der vom Institut veröffentlichten und herausgegebenen Zeitschrift »Revue de l'IFHA«. Dank der Kompetenzen und des savoir-faire der Mitarbeiterinnen und Mitarbeiter von revues.org steht der wissenschaftlichen Gemeinschaft unserer beiden Länder hier also ein dauerhaftes und kostenloses inhaltliches Angebot zur Verfügung, das durch den Wegweiser »Geschichte machen in Frankreich« noch einmal bereichert wird.

Paris und Frankfurt a. M., im Juli 2014 


\section{NOTES}

1. Falk Bretschneider, Mareike König (Hg.), Faire de l'histoire en Allemagne: un guide pour les jeunes chercheurs français, Scholar Guide 1 (2011), http://www.perspectivia.net/content/ publikationen/scholar-guide/histoire-en-allemagne. Alle Links wurden am 12.7.2014 überprüft.

2. Histoire/Geschichte, 3 Bde. hg. von Nathan in Frankreich und Klett in Deutschland: Bd. 1 = L'Europe et le monde de l'antiquité à 1815/Europa und die Welt von der Antike bis 1815 (2011), Bd. 2 = Europa und die Welt vom Wiener Kongress bis 1945/L'Europe et le monde du Congrès de Vienne à 1945 (2008), Bd. 3 = Europa und die Welt seit 1945/L'Europe et le monde depuis 1945 (2006). Vgl. die Homepages von Nathan: http://enseignants.nathan.fr/collection/manuel-francoallemand und von Klett: http://www.klett.de/produkt/isbn/978-3-12-416510-7.

3. Die Reihe wird unter der Leitung des Deutschen Historischen Instituts in Paris vorbereitet (http://www.dhi-paris.fr/de/home/print-publikationen/deutsch-franzoesische-geschichte.html) und in Deutschland bei der Wissenschaftlichen Buchgesellschaft (http://www.wbgwissenverbindet.de/shop/ProductDisplay?

urlRequestType=Base\&catalogId=10051\&categoryId=\&productId=187154\&urlLangId=-3\&langId=-3\&top_category=\&parent_category_rn und in Frankreich bei den Presses Universitaires du Septentrion (http://www.septentrion.com/ fr/collections/histoirefrancoallemande/) veröffentlicht.

4. Deutsches Historisches Institut Paris, http://www.dhi-paris.fr/de/home.html.

5. Centre Marc Bloch, http://www.cmb.hu-berlin.de.

6. Institut français d'histoire en Allemagne, http://www.ifha.fr/de.

7. Liste der integrierten Studiengänge in Geschichte: http://www.dfh-ufa.org/de/studium/ studienfuehrer/mode/liste/fr/18/typ/11/pointer/0/; Liste der Deutsch-Französischen Doktorandenkollegs: http://www.dfh-ufa.org/uploads/media/Tableaux_DFDK_CDFA_2014.pdf.

8. Siehe zu den aktuellen Herausforderungen einer Dissertation in den Geistes- und Sozialwissenschaften etwa das Buch: Moritz Hunsmann, Sébastien Kapp (Hg.), Écrire une thèse en sciences sociales, Paris 2013, http://editions.ehess.fr/ouvrages/ouvrage/devenir-chercheur.

9. Vgl. dazu auch den Themenschwerpunkt "Ecrire les sciences sociales, un enjeu pour les jeunes chercheurs« im Newsletter Nr. 69 (Februar 2014) der EHESS, http://lettre.ehess.fr/6991.

10. Die derzeitige Flut von Bilanzen und Selbstbefragungen der französischen Historiker und ihrer Disziplin ist sicher ein Zeichen für diese Form einer "Krise« - um einen schon etwas in die Jahre gekommenen Titel von Gérard Noiriel (Sur la »crise« de l'histoire, Paris 1996) aufzugreifen. Siehe u.a. Jean-Claude Ruano-Borbalan (Hg.), L'histoire aujourd'hui, Paris 1999; Christian Delacroix, François Dosse, Patrick Garcia, Nicolas Offenstadt (Hg.), Historiographies. Concepts et débats, Paris 2010, 2 Bde.; Jean-François Sirinelli, Pascal Cauchy, Claude Gauvard (Hg.), Les historiens français à l'œuvre, 1995-2010, Paris 2010; L'histoire saisie par la fiction, Nr. 165 (Mai/ August 2011) der Zeitschrift Le Débat; Christophe Granger (Hg.), À quoi pensent les historiens? Faire de l'histoire au XXI ${ }^{\mathrm{e}}$ siècle, Paris 2013.

11. $\mathrm{Zu}$ den intellektuellen Operationen der Wissensproduktion in den Sozialwissenschaften vgl. Autorenkollektiv: Faire des sciences sociales. Critiquer. Comparer. Généraliser, 3 Bde., Paris 2012. 12. François Hartog, Régimes d'historicité. Présentisme et expérience du temps, Paris 2003.

13. So schon Marc Bloch. Aktueller vgl. Patrick Boucheron, Faire profession d'historien, Paris 2010. 


\section{AUTEURS}

\section{FALK BRETSCHNEIDER}

Dr. Falk Bretschneider ist Maître de conférences an der École des hautes études en sciences sociales (EHESS) und Leiter des Europa-Programms und der deutsch-französischen Forschungskooperation an der Fondation Maison des sciences de l'homme in Paris. Mail: Falk.Bretschneider[at]ehess.fr

\section{MAREIKE KÖNIG}

Dr. Mareike König ist Leiterin der Bibliothek und der Abteilung 19. Jahrhundert am Deutschen Historischen Institut Paris sowie Leiterin der Redaktion der deutschsprachigen Blogplattform de.hypotheses.org.

Mail: mkoenig[at]dhi-paris.fr

\section{PIERRE MONNET}

Prof. Dr. Pierre Monnet ist Directeur d'études an der École des Hautes Etudes en Sciences Sociales (EHESS) und leitet seit 2011 das Institut Français d'Histoire en Allemagne in Frankfurt am Main. Mail: Monnet[at]ehess.fr 